\title{
Imaging CSF Shunts and their Complications
}

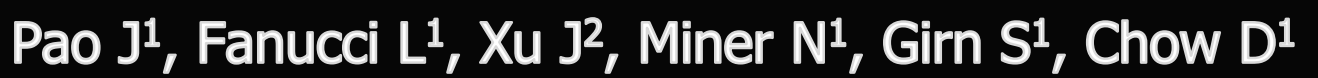

${ }^{1}$ Department of Radiological Sciences, ${ }^{2}$ Department of Neurological Surgery

UC Irvine Medical Center, Orange, CA

\section{CSF Physiology}

- Choroid plexus produces 400-600 mL per day of cerebrospinal fluid (CSF), with $150 \mathrm{~mL}$ present at any given time $(125 \mathrm{~mL}$ subarachnoid $+25 \mathrm{~mL}$ intraventricular)

\section{CSF absorbed by arachnoid villi and lymphatics}

Monro-Kellie doctrine: a dynamic pressure-volume equilibrium exists within the rigid skull between the brain parenchyma, blood, and CSF

- Hydrocephalus ( $\uparrow C S F$ volume) alters this intracranial pressurevolume equilibrium. Examples:

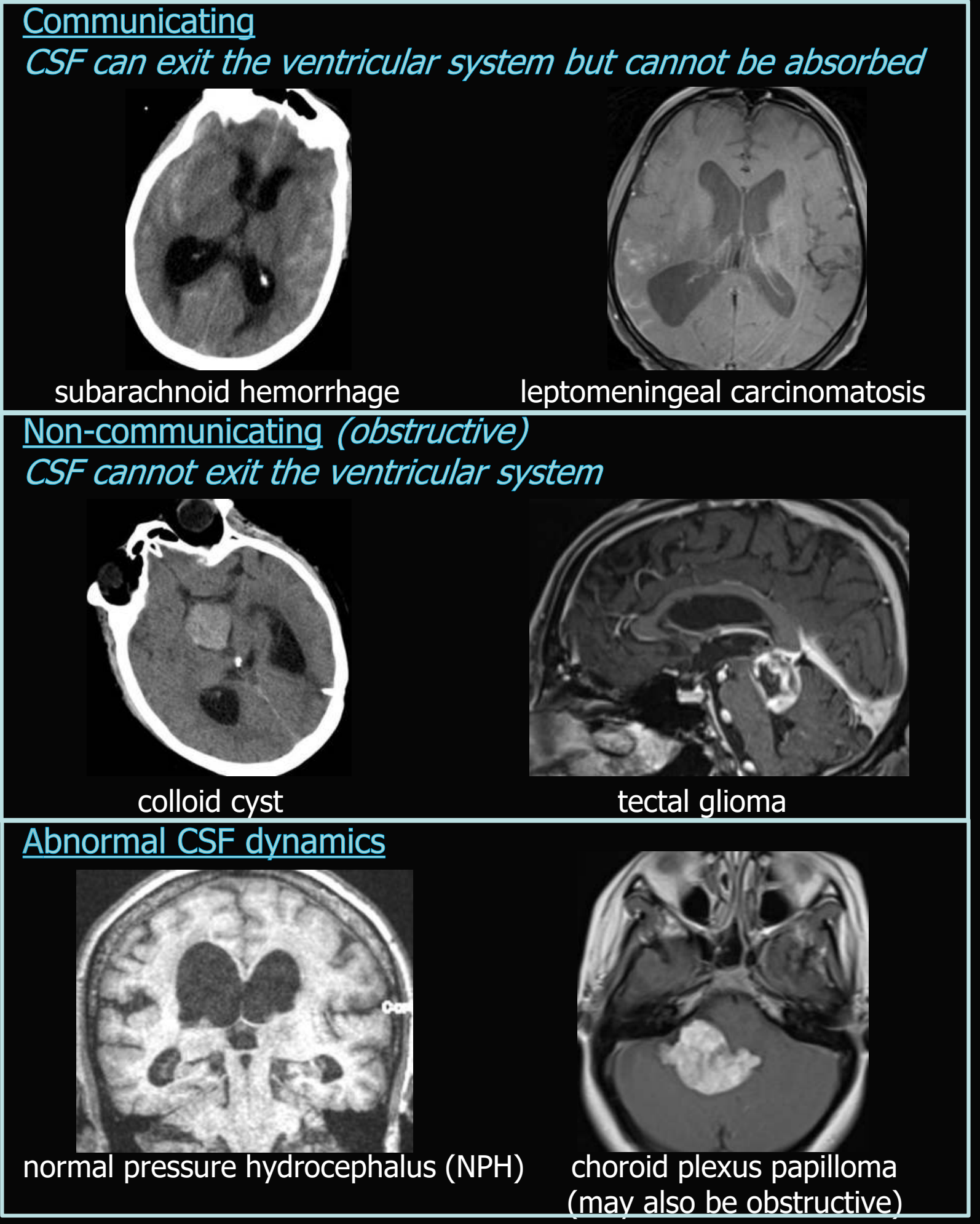

CSF Shunts

- CSF shunts attempt to restore the pressure-volume equilibrium Fixed shunt valve (FSV) - fixed flow rate

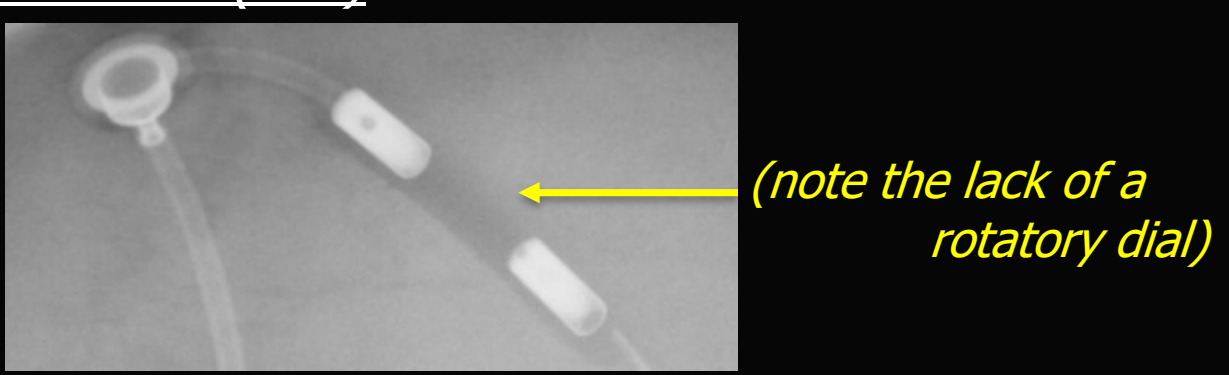

Programmable shunt valve (PSV) - adjustable flow rate using an external codified magnetic tool; ideal for NPH, complex hydrocephalus (Dandy-Walker, Chiari)

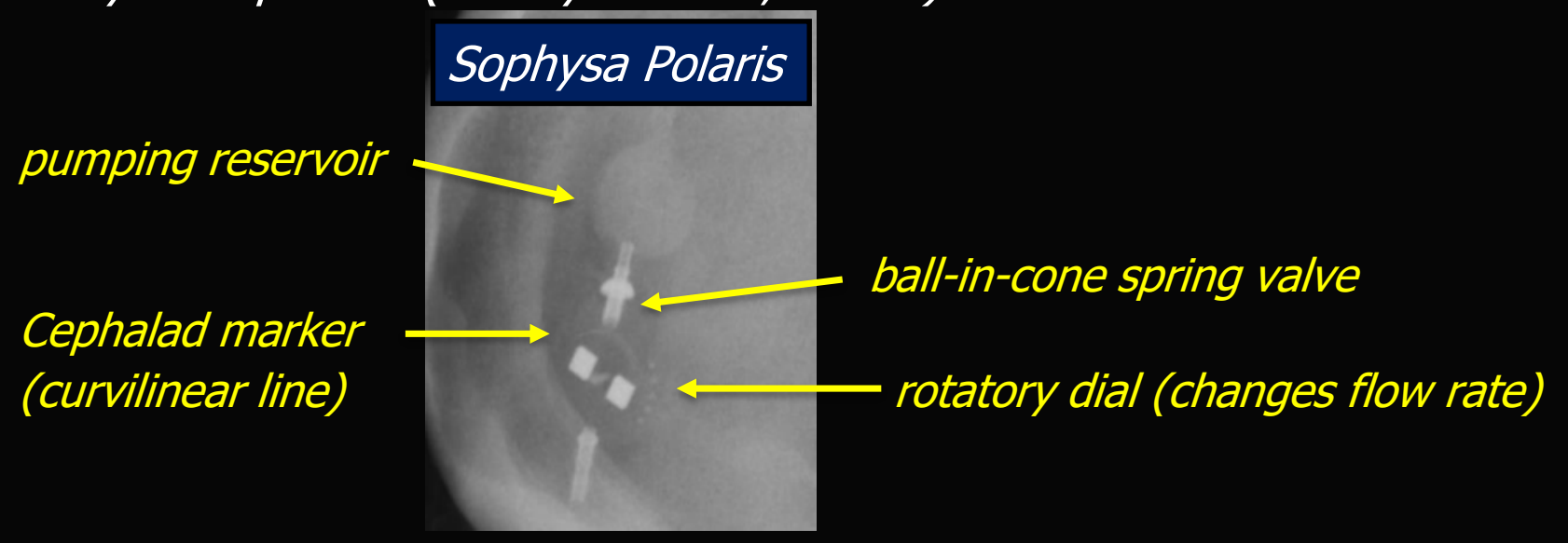

*Each PSV manufacturer has a unique rotatory dial and radiopaque markers (refer to user guide). [Aesculap PSV not shown]

Codman Certas

\section{Shunt courses}

- Ventriculoperitoneal (VP)

- Ventriculoatrial (VA)

- Ventriculopleural (VPL)

Imaging evaluation of shunts

- XR shunt series: AP+lateral views along shunt course

Assess shunt integrity (fracture, disconnection, kinking)

- NM scintigraphy: $\leq 0.4 \mathrm{~mL}\left[{ }^{99 \mathrm{~m} T c-p e r t e c h n e t a t e,}{ }^{99 \mathrm{mT}} \mathrm{T}\right.$-DTPA, or ${ }^{111} \mathrm{In}$ DTPA (0.25-1.5 mCi)] into reservoir, supine position, obtain initial flow and delayed planar images

Differentiate proximal/distal limb obstruction, locate site of leak or obstruction

- US: targeted evaluation

Evaluate for loculations/pseudocyst

CT: cross-sectional view of area of interest

Assess ventricle size, complications along shunt course

- MR: single-shot T2 of ventricles

Assess ventricle size without radiation, limited evaluation of shunt valve itself (susceptibility artifact)

Potential MRI interactions with shunts

\begin{tabular}{|r|r|r|r|}
$\begin{array}{r}\text { Shunt } \\
\text { type }\end{array}$ & $\begin{array}{r}\text { MR field } \\
\text { strength* }\end{array}$ & Performance & $\begin{array}{r}\text { Screening radiograph } \\
\text { after MR exam }\end{array}$ \\
\hline FSV & $\begin{array}{r}\text { Conditional at } \\
1.5 / 3 T\end{array}$ & Not affected & Not required \\
\hline PSV & $\begin{array}{r}\text { Conditional at } \\
1.5 / 3 T\end{array}$ & $\begin{array}{r}\text { Potential } \\
\text { adjustment of } \\
\text { flow rate }\end{array}$ & $\begin{array}{r}\text { Recommend radiograph } \\
\text { (or interrogation with magnet } \\
\text { device) to check settings }\end{array}$ \\
\hline
\end{tabular}

*Refer to manufacturer guide for spatial gradient magnetic field and

specific absorption rate limits. Do not place local transmit coils over valve.

${ }^{1}$ The Sophysa Polaris PSV has not demonstrated inadvertent valve setting

changes by high magnetic field strengths of up to 3T, although the

similarly appearing Sophysa SM8 and other PSVs may be altered.

CSF shunt complications

- Approximately $1 / 3^{\text {rd }}$ of all shunts fail within 1 year of placement
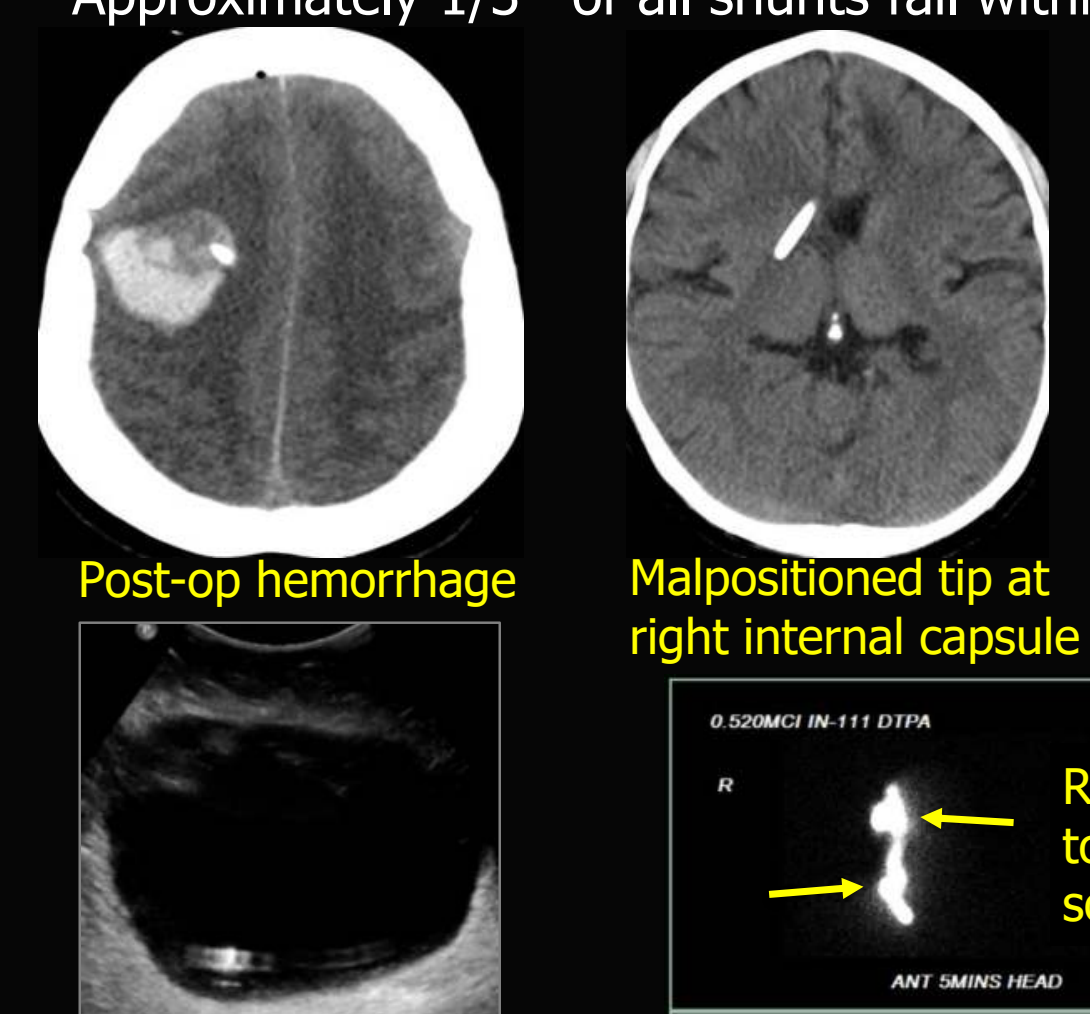

ght internal capsule

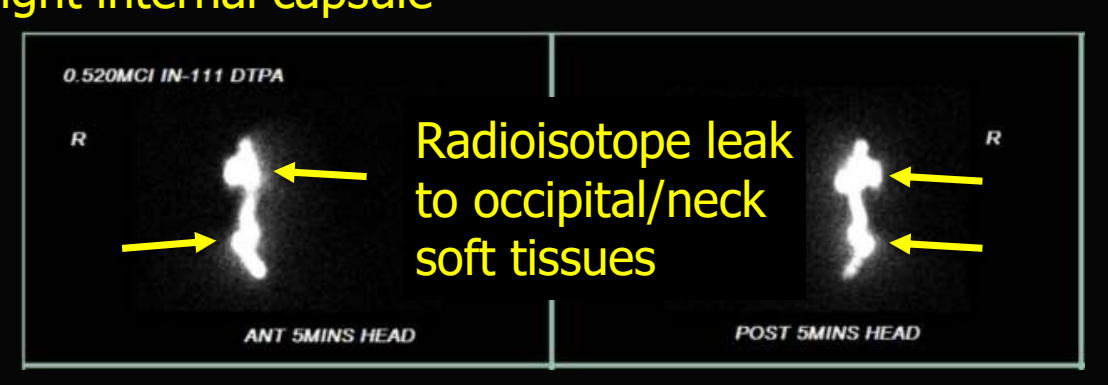

CSF peritoneal pseudocyst

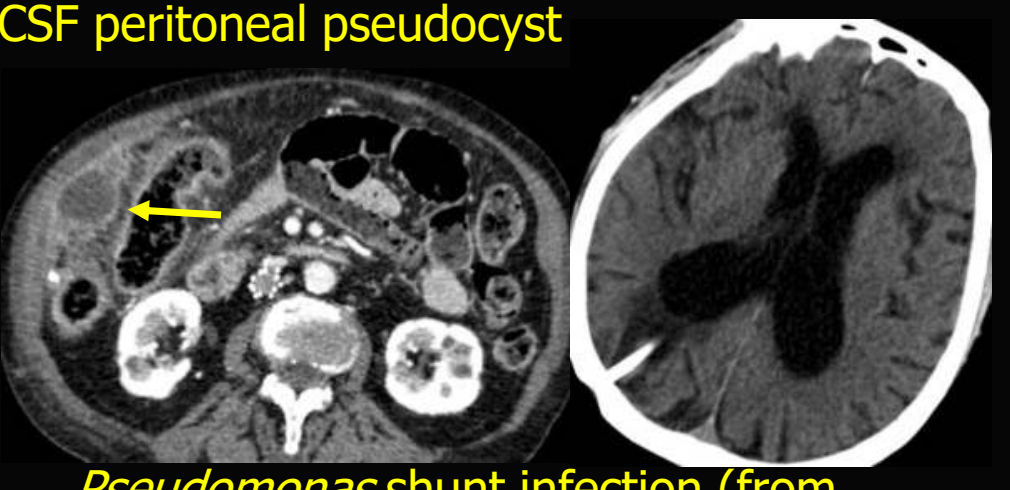

Pseudomonas shunt infection (from

RUQ abscess, edema along shunt tract)

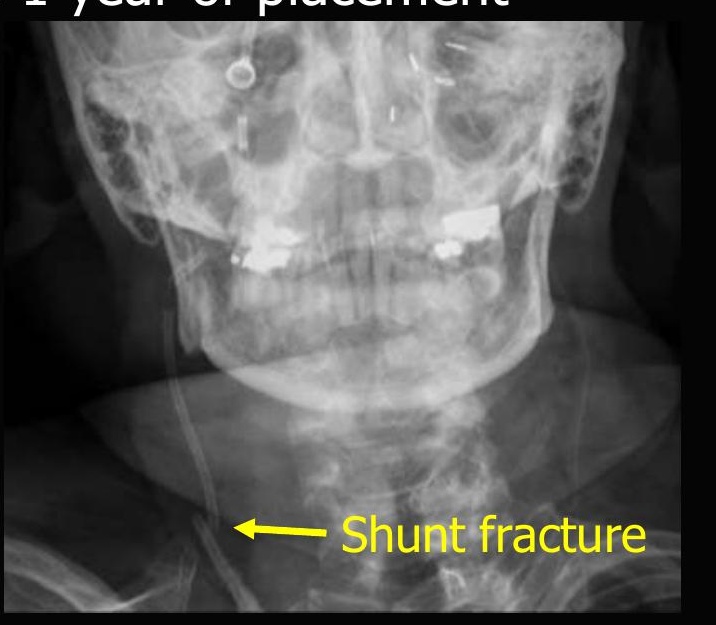

Radioisotope leak occipital/neck

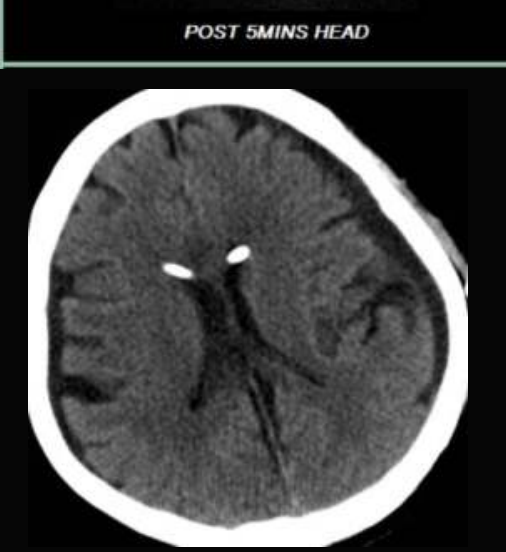

Overshunting (slit-like ventricles + new subdural hygromas)
Other complications: shunt migration, kinking, ventricular loculation, ascites (VP), pleural effusion (VPL), pulmonary arterial hypertension (VA), thromboembolic disease (VA), tricuspid valve injury (VA)

Browd SR, Ragel BT, Gottfried ON, Kestle JR: Failure of cerebrospinal fluid shunts: part I: Obstruction and mechanical failure. $P$ ediatr $N$ eurol $3403-022006$

Browd SR, Gottfried ON, Ragel BT, Kestle JR: Failure of cerebrospinal fluid shunts: part II: overdrainage, loculation, and abdomina complicentions Peditr,

Wallace, A.N., McConathy, J., Menias, C.O. et al, Imaging evaluation of CSF shunts. AJR Am J Roentgenol. 2014;202:38-5 Lollis SS, Mamourian AC, Vaccaro TJ, Duhaime AC. Programmable CSF shunt valves: radiographic identification and interpretation. AJNR 2010; 31:1343-1346

Costello JE, Rasser, UA. An Updace on Programmable CSF Shunt Valves: Identification, MR Imaging Safety, and Potentia 